\title{
Arterial stiffness and cardiovascular risk
}

\author{
Jae-Geun Lee ${ }^{1}$ and Seung-Jae Joo ${ }^{1,2}$
}

${ }^{1}$ Department of Internal Medicine, Jeju National University Hospital, Jeju; ${ }^{2}$ Department of Internal Medicine, Jeju National University School of Medicine, Jeju, Korea

Received: March 31, 2019 Accepted: April 11, 2019

\author{
Correspondence to \\ Seung-Jae Joo, M.D. \\ Department of Internal \\ Medicine, Jeju National \\ University School of Medicine, \\ 15 Aran 13-gil, Jeju 63241, Korea \\ Tel: +82-64-717-1701 \\ Fax: +82-64-725-2593 \\ E-mail: sejjoo@jejunu.ac.kr \\ https://orcid.org/0000-0002- \\ 4322-0703
}

\section{See Article on Page 539-548}

The screening and management of moderate-to-high risk groups for cardiovascular (CV) events is important to reduce mortality, morbidity, and the socioeconomic burden associated with cardiovascular diseases (CVDs). Future CV events have traditionally been predicted using an algorithm that combines conventional atherosclerotic risk factors. However, vascular biomarkers such as carotid ultrasonography, the ankle-brachial index, arterial stiffness, central hemodynamics and wave reflections, endothelial function, and circulating biomarkers related to vascular wall biology are also suggested for the primary and secondary prevention of CV events [1].

Arterial stiffness is basically a disease of the arterial media; it is related to normal or accelerated aging. Indeed, as Thomas Sydenham said, "A man is as old as his arteries." Other atherosclerotic risk factors such as hypertension, diabetes, dyslipidemia, or smoking can change the mechanical properties of the arterial wall, leading to stiffness. A number of invasive and non-invasive methods and indices have been developed to estimate arterial stiffness; among them, pulse wave velocity (PWV) is the most commonly used technique to measure arterial stiffness. PWV is the speed of an arterial pulsation when it is propagating through the arterial tree.
Measurement of the carotid-femoral pulse wave velocity (cfPWV) is a popular and validated method, especially in Western countries. Its value in improving the prediction of $\mathrm{CV}$ events beyond conventional risk factors was shown in a meta-analysis of prospective observational data [2]; cfPWV was a predictor of coronary heart disease, stroke, and CV events, even after adjusting for conventional risk factors such as sex, age, systolic blood pressure (BP), serum cholesterol level, serum high density lipoprotein cholesterol (HDL-C) level, smoking status, diabetes, and antihypertensive medications. According to 2018 European Society of Cardiology/European Society of Hypertension [3] and 2018 Korean Society of Hypertension (KSH) guidelines for the management of arterial hypertension [4], a cfPWV > $10 \mathrm{~m} / \mathrm{sec}$ is considered to indicate hypertension-mediated organ damage.

To be widely used as a screening tool in the general population, a test should be simple, convenient, reproducible, and operator-independent. Although measurement of the cfPWV is a reference technique to evaluate arterial stiffness, the required exposure of the inguinal area and technical experience needed for carotid and femoral pulse recording mean that the cfPWV is mainly used in research settings rather than daily clinical practice. In this regard, the brachial-ankle pulse wave velocity (baPWV) may be an alternative 
index for arterial stiffness. Measurement of the baPWV requires wrapping BP cuffs on four extremities; thus, it is a suitable method for estimating arterial stiffness in a routine clinical setting or population-based study [5]. The proof of concept, prospective validation, and incremental value for predicting future CV events has been established with ease of use [1]. A meta-analysis of prospective studies of Japanese individuals without CVD showed that a higher baPWV was associated with a higher risk of CVD, and the addition of the baPWV to a patient's Framingham risk score (FRS) improved the efficacy for predicting CVD [6]. The proposed cut-off baPWV value to define a stiff artery is $18 \mathrm{~m} / \mathrm{sec}$ [7]. According to $\mathrm{KSH} 2018$ guidelines for the management of arterial hypertension, a baPWV $>18 \mathrm{~m} / \mathrm{sec}$ is considered to indicate subclinical target organ damage in hypertension [4]. However, the application of the baPWV has been mainly confined to Japan and other East Asian countries. The clinical utility, clinical outcomes, and cost-effectiveness of the baPWV have not been fully validated in Western countries. Calculating the distance of wave propagation using a height-based formula is another issue to be validated in diverse ethnic groups [1].

The usefulness of the baPWV as a vascular marker could be shown through a comparison with multivariate risk-predicting systems. In the latest issue of the Korean Journal of Internal Medicine, Rhee et al. [8] reported a correlation between baPWV and four different risk-scoring systems. They compared baPWV not only with the original FRS but also the revised FRS, Adult Treatment Panel III revised FRS or generalized FRS, and the recently proposed American College of Cardiology/American Heart Association CVD risk equation, whose outcome parameters include stroke as well as coronary heart disease. They did not present a cut-off value that classified high-risk groups for future CV events, but the possible prognostic role of baPWV as a vascular biomarker was shown by proving a significant correlation with the risk scores from these four risk-prediction algorithms. They also showed that the correlation was stronger in women than in men, implying better performance of the baPWV in women for predicting CV risk in a healthy population.

Sex differences in arterial stiffness are controversial, and diverse views have been reported. In the study of Rhee et al. [8], the baPWV was higher in women than in men; however, because the women were older than the men, and age is one of the most powerful determining factors for the baPWV, this result was expected. In a Japanese study, the baPWV was lower in women than in men until age 60 and then became similar over the age of 60 ; moreover, the coefficient of the effect of age on baPWV was larger in women than in men [9]. The correlation coefficients between baPWV and other conventional CV risk factors (e.g., mean $\mathrm{BP}$, body mass index, total cholesterol level, HDL-C level, and triglyceride level) were also higher in women than in men, in accordance with the findings of Rhee et al. [8]. The mechanism underlying the reported sex differences in the correlation power between baPWV and CV risk score is unknown. Being male is an important determinant of one's CV risk score, and it may weaken the role of other risk factors in the algorithms.

On the other hand, a Brazilian population-based study showed that the slope of the age-related increase in cfPWV was similar between men and women, and CV risk factors made this slope steeper without sex differences, although the cfPWV of men was higher for all age intervals after adjusting for mean BP [10]. A direct comparison of sex differences in baPWV and cfPWV with aging was possible in one report that used the same equipment to measure the baPWV and cfPWV in the same people [11]. The main results of that study were that the baPWV and cfPWV increased with age, and both values were lower in females than in males. It was also shown that the baPWV was lower in women until age 50; after that, the values in men and women became similar, but the cfPWV was higher in men of all age groups. The increase in baPWV from the third to the seventh decades of life was greater in females, but that in cfPWV was similar in both sexes. These findings suggest that a stronger correlation with CV risk-prediction scores in women may only be applicable in terms of the baPWV; this point should be clarified in future studies.

In a stiff arterial system, the velocities of the backward reflection wave and forward flow are rapid enough for the early return of reflected waves during systole rather than diastole, resulting in an increased central systolic $\mathrm{BP}$ and pulse pressure (PP), decreased PP amplification (the ratio of the peripheral to central $\mathrm{PP}$ ), increased left ventricular afterload, and decreased coronary blood flow [7]. These phenomena are more evident in women than 
in men mainly due to their shorter height, smaller large artery diameter, and lower large and small artery elasticity-even though the PWV is similar in both sexes. It was also shown that the aortic characteristic impedance was higher while the total arterial compliance was lower in women, and the interaction of those indices with left ventricular diastolic function or ventricular-arterial coupling was only observed in women [12]; this may explain why heart failure with a preserved ejection fraction is more common in women.

The arteries and heart of women are a deep ocean that must be explored in sex-specific studies to prevent CVD in older females.

\section{Conflict of interest}

No potential conflict of interest relevant to this article was reported.

\section{REFERENCES}

1. Vlachopoulos C, Xaplanteris P, Aboyans V, et al. The role of vascular biomarkers for primary and secondary prevention. A position paper from the European Society of Cardiology Working Group on peripheral circulation: Endorsed by the Association for Research into Arterial Structure and Physiology (ARTERY) Society. Atherosclerosis 2015;241:507-532.

2. Ben-Shlomo Y, Spears M, Boustred C, et al. Aortic pulse wave velocity improves cardiovascular event prediction: an individual participant meta-analysis of prospective observational data from 17,635 subjects. J Am Coll Cardiol 2014;63:636-646.

3. Williams B, Mancia G, Spiering W, et al. 2018 ESC/ESH guidelines for the management of arterial hypertension: the task force for the management of arterial hypertension of the European Society of Cardiology and the European Society of Hypertension: the task force for the management of arterial hypertension of the European Society of Cardiology and the European Society of Hypertension. J Hypertens 2018;36:1953-2041.

4. Korean Society of Hypertension. 2018 Korean Society of Hypertension guideline for the management of arterial hypertension [Internet]. Seoul (KR): Korean Society of Hypertension, c2018 [cited 2019 Apr 10]. Available from: http://www.koreanhypertension.org/reference/guide?mode=read\&idno $=4246$.

5. Munakata M. Brachial-ankle pulse wave velocity: background, method, and clinical evidence. Pulse (Basel) 2016;3:195-204.

6. Ohkuma T, Ninomiya T, Tomiyama H, et al. Brachial-ankle pulse wave velocity and the risk prediction of cardiovascular disease: an individual participant data meta-analysis. Hypertension 2017;69:1045-1052.

7. Tomiyama H, Matsumoto C, Shiina K, Yamashina A. Brachial-ankle PWV: current status and future directions as a useful marker in the management of cardiovascular disease and/or cardiovascular risk factors. J Atheroscler Thromb 2016;23:128-146.

8. Rhee TM, Kim HL, Oh S, et al. Gender difference in the association between brachial-ankle pulse wave velocity and cardiovascular risk scores. Korean J Intern Med 2019;34:539-548.

9. Tomiyama H, Yamashina A, Arai T, et al. Influences of age and gender on results of noninvasive brachial-ankle pulse wave velocity measurement: a survey of 12517 subjects. Atherosclerosis 2003;166:303-309.

10. Baldo MP, Cunha RS, Molina MDCB, et al. Carotid-femoral pulse wave velocity in a healthy adult sample: the ELSA-Brasil study. Int J Cardiol 2018;251:90-95.

11. Jang SY, Ju EY, Huh EH, Kim JH, Kim DK. Determinants of brachial-ankle pulse wave velocity and carotid-femoral pulse wave velocity in healthy Koreans. J Korean Med Sci 2014;29:798-804.

12. Coutinho T, Borlaug BA, Pellikka PA, Turner ST, Kullo IJ. Sex differences in arterial stiffness and ventricular-arterial interactions. J Am Coll Cardiol 2013;61:96-103. 